\title{
ERRATUM
}

\section{Erratum zu: Erfassung von fachspezifischen Problemlöseprozessen mit Sortieraufgaben in Biologie und Physik}

\author{
Torsten Binder ${ }^{1}$ Philipp Schmiemann ${ }^{1} \cdot$ Heike Theyßen ${ }^{2}$ \\ Online publiziert: 4. Februar 2020 \\ ○) Gesellschaft für Didaktik der Physik und Chemie (GDCP), Fachsektion Didaktik der Biologie im VBIO (FDdB im VBIO) und Springer-Verlag GmbH Deutsch- \\ land, ein Teil von Springer Nature 2020
}

\section{Erratum zu:}

\section{ZfDN 2019}

https://doi.org/10.1007/s40573-019-00090-x

Im Beitrag „Erfassung von fachspezifischen Problemlöseprozessen mit Sortieraufgaben in Biologie und Physik" von T. Binder, P. Schmiemann und H. Theyßen (Zeitschrift für Didaktik der Naturwissenschaften, 25/2019, S. 25-42) fehlt ein Hinweis auf die Dissertation von Martin Dickmann, die in der Arbeitsgruppe der Coautorin H. Theyßen entstanden ist. Dementsprechend sind folgende Ergänzungen vorzunehmen:

Der erste Satz im Abschnitt „Interpretations-NutzungsArgumentation für Sortieraufgaben“ (S. 30) muss lauten:
„Die Validitätsargumentation für die Sortieraufgaben erfolgt anhand einer Interpretations-Nutzungs-Argumentation (INA) in Anlehnung an Kane (2013) und Dickmann (2016).“

Die Bildunterschrift zu Abb. 2 (S. 32) muss lauten: „Abb. 2 Interpretations-Nutzungs-Argumentation für Sortieraufgaben (Darstellung nach Dickmann, 2016, S. 75)“

Im Literaturverzeichnis ist die folgende Quelle zu ergänzen: Dickmann, M. (2016). Messung von Experimentierfähigkeiten. Validierungsstudien zur Qualität eines computerbasierten Testverfahrens. Studien zum Physik- und Chemielernen, Bd. 210. Berlin: Logos.

Die Online-Version des Originalartikels ist unter https://doi.org/ 10.1007/s40573-019-00090-x zu finden.

Torsten Binder

torsten.binder@uni-due.de

1 Didaktik der Biologie 2, Universität Duisburg-Essen, Universitätsstraße 2, 45141 Essen, Deutschland

2 Didaktik der Physik, Universität Duisburg-Essen, Universitätsstraße 2, 45141 Essen, Deutschland 